TITLE:

\title{
Increase in carrier mobility of organic ultrathin-film transistor with increasing molecular layers investigated by Kelvin probe force microscopy
}

\section{$\operatorname{AUTHOR}(\mathrm{S}):$}

Miyazaki, Takashi; Kobayashi, Kei; Ishida, Kenji; Hotta, Shu; Horiuchi, Toshikazu; Matsushige, Kazumi; Yamada, Hirofumi

\section{CITATION:}

Miyazaki, Takashi ... [et al]. Increase in carrier mobility of organic ultrathin-film transistor with increasing molecular layers investigated by Kelvin probe force microscopy. JOURNAL OF APPLIED PHYSICS 2005, 97(12): 124503.

\section{ISSUE DATE:}

2005-06-15

URL:

http://hdl.handle.net/2433/39714

\section{RIGHT:}

Copyright 2005 American Institute of Physics. This article may be downloaded for personal use only. Any other use requires prior permission of the author and the American Institute of Physics. 


\title{
Increase in carrier mobility of organic ultrathin-film transistor with increasing molecular layers investigated by Kelvin probe force microscopy
}

\author{
Takashi Miyazaki $^{\text {a) }}$ \\ Venture Business Laboratory, Kyoto University, Kyoto 606-8501, Japan \\ Kei Kobayashi \\ International Innovation Center, Kyoto University, Kyoto 606-8501, Japan \\ Kenji Ishida \\ Department of Electronic Science and Engineering, Kyoto University, Kyoto 615-8510, Japan \\ Shu Hotta \\ Department of Polymer Science and Engineering, Kyoto Institute of Technology, Kyoto 606-8585, Japan \\ Toshihisa Horiuchi and Kazumi Matsushige \\ Department of Electronic Science and Engineering, Kyoto University, Kyoto 615-8510, Japan \\ Hirofumi Yamadab) \\ Department of Electronic Science and Engineering, Kyoto University, Kyoto 615-8510, Japan \\ and Core Research for Evolutional Science and Technology, Japan Science and Technology Agency, \\ Kyoto 615-8510, Japan
}

(Received 7 September 2004; accepted 25 April 2005; published online 20 June 2005)

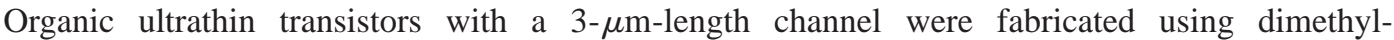
quinquethiophene molecules. The local structures of the films and electric potential profiles across the channels were investigated by Kelvin probe force microscopy with the gate electrode grounded. We found that the local carrier mobility was much higher for the second-layer regions than for the monolayer regions from potential profiles measured while the gate electrode was electrically grounded. However, the macroscopic carrier mobility increased only slightly by increasing the number of molecular layers. One of the possible reasons for this contradiction was the large contact resistance at the source/channel interface, which was apparently observed in the potential profiles. (C) 2005 American Institute of Physics. [DOI: 10.1063/1.1937474]
\end{abstract}

\section{INTRODUCTION}

In the last ten years, organic thin-film transistors (OTFTs) have been intensively studied because of their attractive features such as low-cost and low-temperature processing, printability, and flexibility. ${ }^{1}$ However, the measured field-effect mobility in organic thin films has been relatively low compared to that of inorganic devices, which limits the number of practical applications. ${ }^{2}$ In contrast with OTFTs, the field-effect mobility in organic single crystals can have excellent values as high as $15 \mathrm{~cm}^{2} / \mathrm{V} \mathrm{s}$, ${ }^{3}$ which is much higher than that of hydrogenated amorphous silicon devices. Therefore, such low field-effect mobility values in OTFTs might result from extrinsic influences such as grain boundaries or imperfect interfaces with metal electrodes. Several researchers have demonstrated an improved field-effect mobility by reducing the number of grain boundaries between the source and drain electrodes. ${ }^{4-6}$ Furthermore, it has been reported that accumulated carriers induced by the gate voltage are localized within a few monolayers on the a gate

\footnotetext{
a) Present address: Advanced Electron Devices Laboratory, Toshiba Corporation, Kawasaki 212-8582, Japan; electronic mail: takashi5.miyazaki@toshiba.co.jp

b) Author to whom correspondence should be addressed; electronic mail: h-yamada@kuee.kyoto-u.ac.jp
}

insulator in the OTFTs. ${ }^{7,8}$ Therefore, it is becoming more and more important to understand the transport mechanisms by taking into account the structures of the organic thin films and local electrical properties at the interfaces with metal electrodes in order to improve the field-effect mobility in the OTFTs.

Kelvin probe force microscopy ${ }^{9}$ (KFM) is an ideal tool to simultaneously investigate the local structures and electric potential distributions of electronic devices for the abovementioned purposes. Several research groups including our group have successfully applied this technique to measure the local electric potential profiles across the source and drain electrodes on the OTFTs. ${ }^{10-12}$ We recently demonstrated the operation of a monolayer-channel OTFT and measured the potential profiles across the channel. ${ }^{11}$ In this report, we studied the structural evolution of the dimethylquinquethiophene (M5T) ultrathin films and the potential profiles across the channel by KFM and compared them with the macroscopic transistor characteristics in order to discuss the thickness dependence of carrier transport and the effect of the contact resistance.

\section{EXPERIMENT}

We fabricated a bottom-contact OTFT with M5T molecules ${ }^{13}$ whose chemical structure is shown in Fig. 1. A 


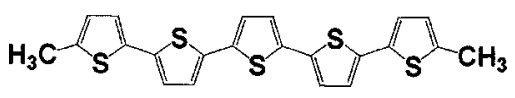

FIG. 1. Chemical structure of M5T molecule.

highly doped silicon wafer with a thermally grown 300-nm-thick silicon oxide layer was used as the substrate. Two 20-nm-thick gold electrodes separated with a gap of $3 \mu \mathrm{m}$ were fabricated on the substrate using the lift-off technique. A chromium layer was deposited prior to the gold deposition as an adhesion promoter. The substrate with electrodes was cleaned using a UV-ozone cleaner at room temperature. M5T molecules were then uniformly deposited onto the substrate by vacuum evaporation. The vacuum pressure and the substrate temperature during the deposition were $2.0 \times 10^{-4} \mathrm{~Pa}$ and $80^{\circ} \mathrm{C}$. The deposition rate was monitored using a quartz-crystal microbalance and it was typically $0.1 \mathrm{~nm} / \mathrm{min}$. We estimated the nominal thickness of the channel region from topographic images obtained by atomic force microscopy (AFM). The deposited M5T films showed a layer-by-layer growth with their molecular axes perpendicular to the substrate surface when prepared under this condition. ${ }^{14}$ The thickness of each molecular layer was about $2.2 \mathrm{~nm}$, thus the nominal thickness of $2.2 \mathrm{~nm}$ corresponded to a complete monolayer.

Topographic imaging and surface-potential mapping by KFM were performed using a scanning probe microscope (JEOL: JSPM-4200) in a vacuum environment (1 $\times 10^{-3} \mathrm{~Pa}$ ) to avoid shielding of the surface potential by the surface water layer. ${ }^{15}$ A Pt-coated Si cantilever (Olympus: AC240TM-B2) was used. While the cantilever was electrically grounded, an ac modulation voltage and a dc feedback voltage from KFM electronics were applied to one of the two electrodes (source). Another gold electrode and silicon substrate were defined as the drain and gate, respectively. The details of the setup were described in a previous paper. ${ }^{11} \mathrm{We}$ could perform KFM measurements on the OTFT while we applied a drain voltage as high as $5 \mathrm{~V}$ since a large drain voltage caused an unstable tip-sample distance regulation. A semiconductor characterization system (Keithley: SCS-4200) was used to measure the TFT characteristics under a vacuum environment $\left(6 \times 10^{-3} \mathrm{~Pa}\right)$.

\section{RESULTS AND DISCUSSION}

M5T films between electrodes showed a layer-by-layer growth as the amount of the deposited molecules increased. ${ }^{11}$ The M5T grains started to grow from the electrode edges during the initial stage of the deposition and then expanded toward the center of the gap. A further increase in the deposited molecules resulted in a full coverage over the gap (complete monolayer) which led to the second layer. The growth of the second layer usually started at the electrode edges, but once in a while, it began in the middle of the gap and resulted in island structures. Figures 2(a) and 2(b) show a topographic image and a surface-potential image of the film as thick as 1.5 monolayers (MLs). These images were obtained while the drain electrode $\left(V_{d}\right)$ and the gate electrode $\left(V_{g}\right)$ were maintained at -1 and $0 \mathrm{~V}$, respectively. In Fig. 2(a), we can see that the gap was fully covered with the first layer on

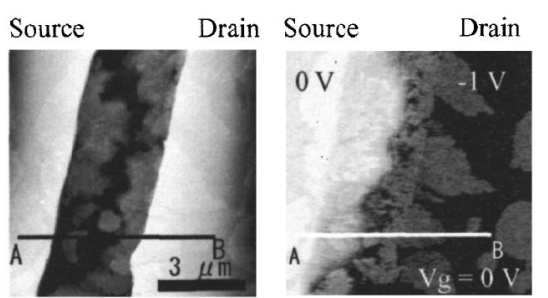

(a)

(b)

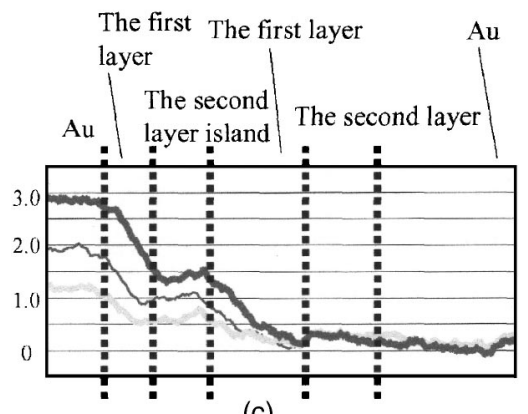

(c)

FIG. 2. Topographic image (a) and KFM image (b) of M5T channel with the second-layer islands. The applied drain voltage $\left(V_{d}\right)$ was -1 V. (c) Potential profiles along the $A-B$ line indicated in (a) were measured at $V_{d}$ voltages of $-1,-2$, and $-3.0 \mathrm{~V}$.

which the second layer formed island structures. Figure 2(c) shows the surface-potential profiles obtained along the $A-B$ line indicated in Figs. 2(a) and 2(b). In Fig. 2(c), the surface potential gradually dropped in the first layer while it was almost constant on the second layer independently of $V_{d}$. If the second layer was electrically insulated from the first layer, the potential profile should discontinuously change at the edges of the second-layer island. However, the potential profiles in Fig. 2(c) were continuous at the edges, thus we postulate that the second layers serve as a carrier path in the channel. Since the slope of the potential profile is proportional to its electrical resistance, it is clear that the conductivity of the two-monolayer region is significantly improved. In general, the sheet conductivity $(\sigma)$ is expressed as

$$
\sigma=N e \mu,
$$

where $N, e$, and $\mu$ are the carrier density per unit area, the elementary charge, and the carrier mobility, respectively. Since these measurements were performed at $V_{g}=0 \mathrm{~V}$, we assume that the field-induced carrier densities for the monolayer region and the second-layer-island regions were almost the same. The abrupt increase in the conductivity could then be attributed to an increase in the local mobility. Furthermore, we performed the same measurements for the M5T layers with different coverages, as shown in Fig. 3. The nominal thicknesses of these M5T films were 2.8 MLs and more than three MLs. Figures 3(c) and 3(f) show the measured potential profiles across the channel. We can see that the slope of the profile on the channel was almost flat, which strongly suggests a further improvement in the local mobility. This is in very good agreement with the recent report by Dinelli et al. in which they concluded that the second layer was crucial for charge transport in the OTFTs. ${ }^{8}$ On the other hand, a large potential drop was observed at the interface with the source electrode as shown in Figs. 3(c) and 3(f), 


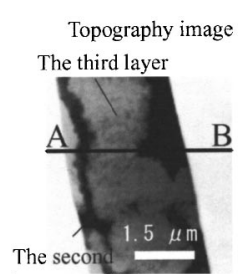

(a)

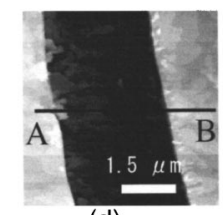

(d)

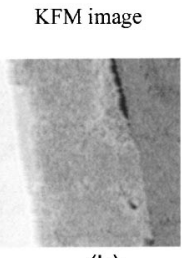

(b)

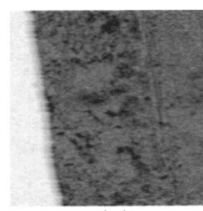

(e)
Surface potential profile

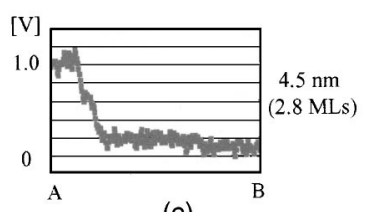

(c)

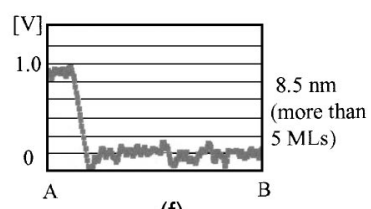

(f)
FIG. 3. Topographic image (a) and (d), KFM image (b) and (e), and potential profile (c) and (f) of M5T channels whose nominal thicknesses were 2.8 MLs and more than three MLs, respectively.

which indicates a large contact resistance at the interface between the channel and the source electrode. We measured the potential profiles for the channel with a thickness greater than three MLs with various gate voltages while the drain voltage was maintained at $-2 \mathrm{~V}$. The result is shown in Fig. 4. These potential profiles seem to be almost the same, which suggests that the contact resistance was extremely greater than the channel resistance. Figure 5(a) shows the drain current $\left(I_{d}\right)$-drain voltage $\left(V_{d}\right)$ curves measured with various gate voltages $\left(V_{g}\right)$ for M5T multilayers as thick as three MLs. In Fig. 5(a), the electrical resistance is clearly modulated with the gate voltage in the same range as Fig. 4 (between 0 and $-30 \mathrm{~V}$ ). The gate effect is not clear when $V_{d}$ was less than $2 \mathrm{~V}$, in which the potential profiles were taken by the KFM. However, we believe that the contact resistance was strongly modulated by the gate voltage in this OTFT although it is still large. In fact, the calculated contact resistance value $\left(R_{S}\right)$ based on Jain's model, where $R_{s}$ is treated as a constant, was not self-consistent. ${ }^{16}$ In this model, the resistance between the source and the drain is expressed as

$$
\frac{1}{g_{d}}=R_{s}+\frac{L}{Z \mu C_{i}\left(V_{g}-V_{0}\right)},
$$

where $Z$ and $L$ are the channel width and length, respectively, $C_{i}$ is the gate capacitance per unit area, $g_{d}$ is the channel

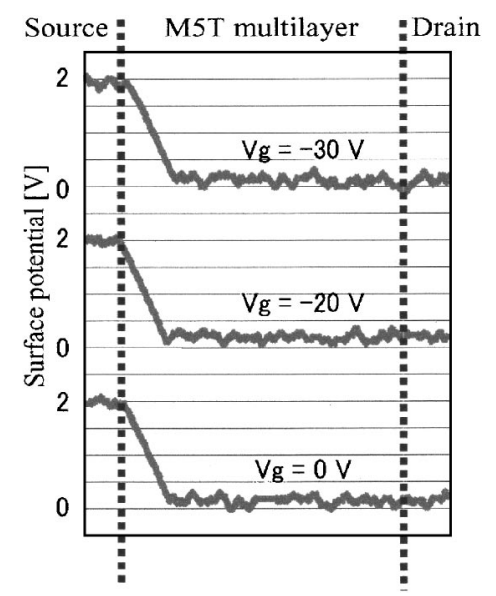

FIG. 4. Potential profiles of M5T multilayer channel measured with various gate voltages $\left(V_{g}\right)$ while $V_{d}$ was fixed at $-2 \mathrm{~V}$.
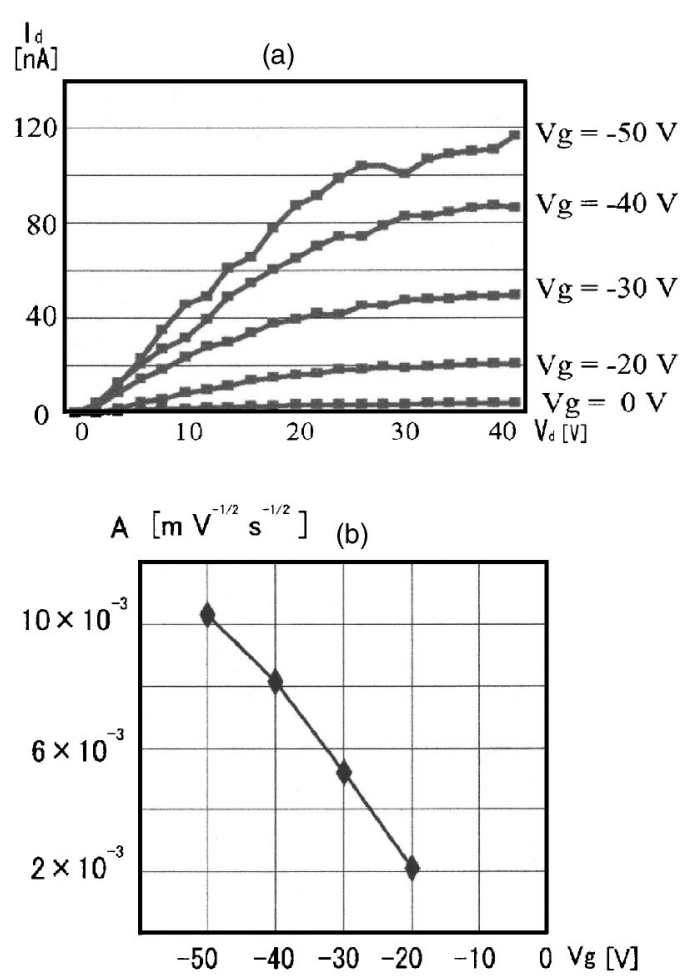

FIG. 5. (a) Transistor characteristics of M5T TFT whose nominal thickness was three MLs. (b) Plot of variable $A$ for various $V_{g}$ voltages obtained from (a).

conductance, $\mu$ is the carrier mobility, and $V_{0}$ is the threshold voltage. In our case, $L$ is $55 \mu \mathrm{m}, Z$ is $3 \mu \mathrm{m}$, and $C_{i}$ is $112 \mu \mathrm{F} . \mu$ and $V_{0}$ are obtained by plotting the following variable $A$ as a function of $V_{g}$ :

$$
A=\frac{g_{d}}{\sqrt{g_{m}}} \sqrt{\frac{L}{Z} \frac{V_{d}}{C_{i}}}=\sqrt{\mu}\left(V_{g}-V_{0}\right),
$$

where $g_{m}$ is transconductance. The values of $g_{d}$ and $g_{m}$ are obtained from the transistor characteristics shown in Fig. 5(a). Figure 5(b) shows the $A-V_{g}$ plots. The slope and the intercept with the $V_{g}$ axis represent $\sqrt{\mu}$ and $V_{0}$, respectively. In Fig. 5(b), the plot is almost linear, so we regarded the carrier mobility as a constant in the calculation for $R_{s}$. The calculated values are shown in Table I. The $R_{s}$ value varies from 50 to $320 \mathrm{M} \Omega$. Moreover, the contact resistance at $V_{g}$ $=-30 \mathrm{~V}$ was lower than one-third of the channel resistance, which contradicts the potential profiles shown in Fig. 4. Therefore, it is clear that the contact resistance was modulated by the gate voltage. The negative gate voltage lowered the contact resistance, then the total conductance increased. Since the reduced contact resistance was still much higher than the channel resistance and the gate effect was not prominent at the low $V_{d}$, the potential profiles in Fig. 4 were almost the same. The origin of this gate modulation is un-

TABLE I. The total resistances and the calculated $R_{s}$ values at various gate voltages.

\begin{tabular}{ccccc}
\hline \hline Gate voltage $(\mathrm{V})$ & -20 & -30 & -40 & -50 \\
Channel resistance $(\mathrm{M} \Omega)$ & 1000 & 500 & 330 & 220 \\
Contact resistance $(\mathrm{M} \Omega)$ & 320 & 160 & 100 & 50 \\
\hline \hline
\end{tabular}


clear, but the energy barrier formed at the channel/source electrode interface ${ }^{17}$ or grain boundaries near the interface ${ }^{18}$ might be lowered by the gate voltage.

Finally, we estimated the macroscopic carrier mobility in order to discuss the effect of the film thickness on the transistor character. The macroscopic carrier mobility was 7.5 $\times 10^{-4} \mathrm{~cm}^{2} / \mathrm{V}$ s from Fig. 5(b). On the other hand, it was $1.2 \times 10^{-4} \mathrm{~cm}^{2} / \mathrm{V}$ s for the M5T single-layer transistor. ${ }^{11}$ It is also reported that the macroscopic carrier mobility of a 500 -nm-thick channel was $5.0 \times 10^{-4} \mathrm{~cm}^{2} / \mathrm{V} \mathrm{s} .{ }^{19}$ The macroscopic carrier mobility was improved by increasing the channel thickness from a single layer while the increase in the film thickness of more than two monolayers produced no improvement in the macroscopic carrier mobility. These results are in agreement with the previous reports that the field-induced carriers are confined within the first two layers. ${ }^{8,20,21}$ Such a limited carrier distribution was also confirmed by the surface-potential profile shown in Fig. 3(f). In Fig. 3(f), the growth of the third layer or upper layers caused no change, which indicates that there is almost no fieldinduced carrier above the third layer. Moreover, this also agrees with our previous KFM measurements of the M5T films on metals. ${ }^{22,23}$ These experiments showed that the differences in the surface potential between the adjacent layers became smaller by increasing the number of layers, and thus, the surface potential on the M5T films was saturated on the second layer. However, the increase in the macroscopic carrier mobility is much lower than expected from the KFM results. It is thought that the large contact resistance almost spoiled the effect of the improved local mobility. Fabrication of the channel layers with fewer grain boundaries and a lower contact resistance and stable KFM measurements independent of drain and gate voltages are desired for further studies.

\section{CONCLUSION}

In this study, we found that the conductivity of the M5T channel abruptly increased by the growth of the second layer based on KFM measurements. The probable reason for the abrupt increase in the conductivity was the increase in the local mobility. In contrast, the macroscopic carrier mobility for the M5T transistor with the nominal thickness of about three MLs was only about six times greater than that in the monolayer-channel transistor measured in a previous experi- ment. This contradiction was probably due to the large contact resistance which was observed in the potential profiles obtained from the KFM measurements.

\section{ACKNOWLEDGMENTS}

This work was supported by a Grant-in-Aid for Scientific Research and Grants for Regional Science and Technology Promotion from the Ministry of Education, Culture, Sports, Science and Technology of Japan, and by Core Research for Evolutional Science and Technology (CREST) of the Japan Science and Technology Corporation (JST). The authors would also like to acknowledge the Kyoto University Venture Business Laboratory Project.

${ }^{1}$ Z. Bao, J. A. Rogers, and H. E. Katz, J. Mater. Chem. 9, 1895 (1999).

${ }^{2}$ Y. Y. Lin, D. J. Gundlach, and T. N. Jackson, IEEE Electron Device Lett. 18, 606 (1997).

${ }^{3}$ V. C. Sundar, J. Zaumseil, V. Podzorov, E. Menard, R. L. Willett, T. Someya, M. E. Gershenson, and J. A. Rogers, Science 303, 1644 (2004).

${ }^{4}$ G. Horowitz and M. E. Hajlaoui, Synth. Met. 122, 185 (2001).

${ }^{5}$ T. W. Kelley and C. D. Frisbie, J. Phys. Chem. B 105, 4538 (2001).

${ }^{6}$ T. Minari, T. Nemoto, and S. Isoda, J. Appl. Phys. 96, 769 (2004).

${ }^{7}$ G. Horowitz, M. E. Hajlaoui, and R. Hajlaoui, J. Appl. Phys. 87, 4456 (2000).

${ }^{8}$ F. Dinelli, M. Murgia, P. Levy, M. Cavallini, and F. Biscarini, Phys. Rev. Lett. 92, 116802 (2004).

${ }^{9}$ M. Nonnenmacher, M. P. O. Boyle, and H. K. Wickramasinghe, Appl. Phys. Lett. 58, 2921 (1991).

${ }^{10}$ L. Burgi, H. Sirringhaus, and R. H. Friend, Appl. Phys. Lett. 80, 2913 (2002).

${ }^{11}$ T. Miyazaki, K. Kobayashi, K. Ishida, S. Hotta, T. Horiuchi, H. Yamada, and K. Matsushige, Jpn. J. Appl. Phys., Part 1 42, 4852 (2003).

${ }^{12}$ K. P. Puntambekar, P. V. Pesavento, and C. D. Frisbie, Appl. Phys. Lett. 83, 5539 (2003).

${ }^{13}$ S. Hotta and K. Waragai, Adv. Mater. (Weinheim, Ger.) 5, 896 (1993).

${ }^{14}$ K. Umeda, K. Kobayashi, K. Ishida, S. Hotta, H. Yamada, and K. Matsushige, Jpn. J. Appl. Phys., Part 1 40, 4381 (2001).

${ }^{15}$ H. Sugimura, Y. Ishida, K. Hayashi, O. Takai, and N. Nakagiri, Appl. Phys. Lett. 80, 1459 (2002).

${ }^{16}$ S. Jain, IEE Proc., Part I: Solid-State Electron Devices 135, 162 (1988).

${ }^{17}$ A. B. Chwang and C. D. Frisbie, J. Phys. Chem. B 104, 12202 (2000).

${ }^{18}$ I. Kymissis, C. D. Dimitrakopoulos, and S. Purushothaman, IEEE Trans. Electron Devices 48, 1060 (2001).

${ }^{19}$ K. Waragai, H. Akimichi, S. Hotta, and H. Kano, Phys. Rev. B 52, 1786 (1995).

${ }^{20}$ A. Dodabalapur, L. Torsi, and H. E. Katz, Science 268, 270 (1995).

${ }^{21}$ M. Kiguchi, M. Nakayama, K. Fujiwara, K. Ueno, T. Shimada, and K. Saiki, Jpn. J. Appl. Phys., Part 2 42, L1408 (2003).

${ }^{22}$ H. Yamada, T. Fukuma, K. Kobayashi, and K. Matsushige, Appl. Surf. Sci. 188, 391 (2002).

${ }^{23}$ T. Miyazaki, K. Kobayashi, K. Ishida, S. Hotta, T. Horiuchi, H. Yamada, and K. Matsushige (unpublished). 